\title{
Zukunft der Arbeit in Industrie 4.0 - Szenarien aus Forschungs- und Entwicklungsprojekten
}

\author{
Steffen Wischmann und Ernst Andreas Hartmann
}

\subsection{Gestaltungskonzepte für die Zukunft der Arbeit gespiegelt in Forschungs- und Entwicklungsprojekten}

Der hier vorliegende Band ist einerseits eine in sich abgeschlossene, konzeptionell für sich stehende Publikation, zugleich kann er aber auch verstanden werden als zweiter Band zu dem bereits vorliegenden Sammelband ,Zukunft der Arbeit in Industrie 4.0“. ${ }^{1}$

In dieser ersten Publikation wurden grundlegende Perspektiven, Herausforderungen, Technologiefelder sowie Forschungs- und Entwicklungsstrategien für die Zukunft der Arbeit in Industrie 4.0 dargestellt. Über die einzelnen Beiträge aus Wirtschaft, Sozialpartnerorganisationen und Wissenschaft hinweg lassen sich zentrale Thesen dieses ersten Bandes wie folgt zusammenfassen:

- Die Zukunft der Arbeit ist gestaltbar und gestaltungsbedürftig. Es gibt keinen technologischen Determinismus - die Folgen der unter „Industrie 4.0“ subsumierten neuen Technologien für Arbeitswelt und Arbeitsmarkt entstehen nicht unmittelbar aus Merkmalen dieser Technologien selbst, sondern aus den Anwendungs- und Einsatzmodellen für diese Technologien, aus Modellen und Szenarien der Gestaltung von Arbeit auf gesellschaftlicher, organisationaler und individueller, auf den Einzelarbeitsplatz bezogener Ebene.

${ }^{1}$ Botthof, A., \& Hartmann, E. A. (Hrsg.) (2015). Zukunft der Arbeit. Berlin.

S. Wischmann $(\bowtie) \cdot$ E.A. Hartmann

Institut für Innovation und Technik, Steinplatz 1, 10623 Berlin, Deutschland

e-mail: wischmann@iit-berlin.de; hartmann@iit-berlin.de 
- Hinsichtlich dieser Folgen für Arbeitswelt und Arbeitsmarkt besonders bedeutsame Aspekte dieser Anwendungs- und Einsatzszenarien beziehen sich auf die zu Grunde liegenden Organisationsmodelle. Hier können zwei paradigmatische Modelle unterschieden werden. Das eine Modell stellt eine Substitution menschlicher Arbeit durch Technik in den Vordergrund (Automatisierungsszenario). Die Aufgabengestaltung orientiert sich an weitgehender Arbeitsteilung, so werden etwa operative und dispositive Aufgaben in der Regel verschiedenen Beschäftigten zugewiesen. Die Qualifikationsstruktur im Unternehmen tendiert zur Polarisierung: Hoch Qualifizierten auf der einen Seite stehen niedrig Qualifizierte auf der anderen Seite gegenüber; diese Spaltung vergrößert sich in diesem Szenario tendenziell.

- Ein alternatives Modell betrachtet Technik eher als Mittel zur Unterstützung und Verstärkung menschlicher Fähigkeiten (Werkzeugszenario). Die Aufgabenteilung ist hier weniger stark ausgeprägt, operative und dispositive Tätigkeiten werden stärker gemischt, insbesondere in dem Sinne, dass operative Tätigkeiten mit dispositiven Tätigkeitsanteilen angereichert werden. Die Qualifikationsstruktur im Unternehmen ist weniger stark polarisiert, es besteht eher ein allgemeiner Trend zur Höherqualifizierung. Niedriger qualifizierte Tätigkeiten werden entweder durch neue Aufgabenverteilung aufgewertet oder durch Automatisierung obsolet. Das „Füllen von Automatisierungslücken“ mit menschlicher Arbeit findet sich deutlich weniger als im Automatisierungsszenario.

- In engem Zusammenhang mit diesen Organisationsmodellen lassen sich Prinzipien progressiver Arbeitsgestaltung für Industrie 4.0 auf unterschiedlichen Aggregationsstufen formulieren. Ein übergeordnetes Gestaltungsprinzip ist die lernförderliche Arbeitsorganisation. Teilaspekte der lernförderlichen Arbeitsorganisation betreffen zunächst die Vollständigkeit von Arbeitstätigkeiten. Arbeitstätigkeiten sind hierarchisch vollständig, wenn sie anspruchsvolle und Routineaufgaben in angemessenen Anteilen verbinden. Sie sind sequentiell vollständig, wenn planerische, organisierende, ausführende und kontrollierende Tätigkeitsanteile an einem Arbeitsplatz kombiniert sind. Weitere Aspekte der Lernförderlichkeit sind Autonomie - Handlungs- und Entscheidungsfreiräume - sowie Transparenz: Kenntnis über die Ergebnisse der eigenen Arbeit und Kenntnis über andere, verbundene Arbeitsprozesse im Unternehmen.

- Lernförderliche Arbeitsorganisation ist zugleich innovationsförderliche Arbeitsorganisation. Dies hat einmal damit zu tun, dass mit komplexen Aufgaben höhere Kompetenzniveaus der Beschäftigten einhergehen. Das erleichtert sowohl die Wahrnehmung externer Innovationsimpulse (z. B. neue Bearbeitungsverfahren) wie auch die interne Verarbeitung, Umsetzung und Verbreitung dieser Innovationsimpulse. Zweitens erhöht die höhere Lernfähigkeit auch die Wahrscheinlichkeit, dass intern (z. B. im Zuge kontinuierlicher Verbesserungsprozesse) Innovationen erdacht und umgesetzt werden können.

- Die der Industrie 4.0 zu Grunde liegenden Technologien lassen sich beschreiben als verteilte, (semi-)autonome, intelligente und vernetzte cyber-physikalische Systeme. Diese technologischen Grundmerkmale finden sich einerseits in für die Industrie typischen Technologiebereichen (z. B. Produktionssysteme, Robotik, Logistiksysteme), 
aber auch in anderen Wirtschaftszweigen (z. B. autonome Landmaschinen in der Landwirtschaft, Anwendungen der Künstlichen Intelligenz im Dienstleistungsbereich).

- Die technologischen Eigenschaften der cyber-physikalischen Systeme der Industrie 4.0 implizieren besondere Potenziale einer progressiven Arbeitsgestaltung. Dies liegt zunächst in der Mächtigkeit und Plastizität dieser Technologien begründet, was sie für die Umsetzung einer großen Palette möglicher Arbeitsgestaltungsmodelle geeignet erscheinen lässt. Ein besonderer Aspekt der Industrie 4.0 ist der Datenreichtum. Eine Vielzahl dezentraler und autonomer Sensoren erfasst in Echtzeit große Datenmengen, die durch neue Techniken der Datenverarbeitung analysiert und visualisiert werden können. Solche Analyse- und Visualisierungstools sind wichtige Elemente einer Gestaltungsstrategie, die Technik als Verstärker und Unterstützer menschlicher Fähigkeiten begreift.

- Neben diesen Potenzialen bestehen auch Risiken. Industrie 4.0 ist letztlich auch ein Automatisierungskonzept und damit anfällig für grundlegende Probleme der Automatisierung. Ein solches grundlegendes Problem zeigt sich in den sogenannten Automatisierungsdilemmata oder -paradoxien: Durch Automatisierung verschieben sich menschliche Tätigkeiten vom aktiven Steuern der Systeme zur Überwachung automatischer Regelung und zum Einspringen in Situationen, die der Automat nicht beherrscht. Solche Situationen sind tendenziell eher komplex. Mit einer solchen komplexen Situation sehen sich nun Menschen konfrontiert, die aus zwei Gründen nicht gut darauf vorbereitet sind, diese Situationen zu meistern. Erstens hat der Mensch diese Situation nicht selbst herbeigeführt und ist deswegen aktuell nicht „,im Bilde“; dies ist ein Problem des Kurzzeitgedächtnisses. Zweitens fehlt durch die Automatisierung die Übung im aktiven Steuern des Systems und damit degradieren die Fähigkeiten, die zur Systemsteuerung notwendig sind; dies ist ein Problem des Langzeitgedächtnisses, das u. a. durch Simulatortraining adressiert werden kann. Für das Problem des Kurzzeitgedächtnisses - das ,im Bilde sein“ über den aktuellen Systemzustand - gibt es Gestaltungsmethoden für automatisierte Systeme wie etwas das Ecological Interface Design, das Möglichkeiten anbietet, den Menschen auf unterschiedlichen Abstraktionsebenen in der Regelschleife des (semi-)automatischen Systems zu halten.

Diese theoriegeleiteten Überlegungen zur Gestaltung von Arbeit in Industrie 4.0 werden im hier vorliegenden Band bezogen auf ganz konkrete technisch-organisationale Lösungen, wie sie in industrienahen Forschungs- und Entwicklungsprojekten realisiert werden. Diesen Projekten ist der Hauptteil dieses Bandes gewidmet.

\subsection{Szenarien aus der Praxis}

Alle Szenarien, die in den Projekten entwickelt werden, lassen sich mit einem einheitlichen Beschreibungsmodell im Hinblick auf die Implikationen für die Arbeitsgestaltung darstellen. Dieses Beschreibungsmodell umfasst folgende Kategorien: 
1. Bedarf: Wie wird sich das technisch-organisationale Szenario (bspw. eine konkrete Einsatzform von kollaborativen Robotern) auf den Bedarf nach verschiedenen Qualifikationsprofilen (z. B. Facharbeiter, Meister, Ingenieure) auswirken?

2. Hierarchische Vollständigkeit: Wie wird sich das Szenario auswirken hinsichtlich

- Monotonie und komplexen Aufgaben?

- problemlösenden und Optimierungsaufgaben?

- Lernen in der Arbeit?

3. Sequentielle Vollständigkeit: Wie wird sich das Szenario auswirken hinsichtlich

- Planungsaufgaben?

- Kommunikation und Kooperation?

4. Kontrolle und Autonomie: Wie wird sich das Szenario auswirken hinsichtlich

- der Kontrolle des Menschen über seine Arbeitssituation?

- der Selbstbestimmung, den Handlungs- und Entscheidungsspielräumen in der Arbeitssituation?

5. Querschnittliche und gegenstandsspezifische Aspekte: Wie wird sich das Szenario auswirken hinsichtlich

- der Interdisziplinarität in der Arbeitssituation?

- der Bedeutung von IT-Kenntnissen?

In einem einleitenden Kapitel stellen Steffen Wischmann und Ernst Hartmann diese Beschreibungsdimensionen vor und präsentieren eine integrierte Betrachtung der Auswirkungen hinsichtlich dieser Dimensionen über alle Praxisprojekte.

In einem ersten Praxisbeispiel stellen Andreas Bächler, Liane Bächler, Sven Autenrieth, Hauke Behrendt, Markus Funk, Georg Krüll, Thomas Hörz, Thomas Heidenreich, Catrin Misselhorn und Albrecht Schmidt Systeme zur Assistenz und Effizienzsteigerung in manuellen Produktionsprozessen der Industrie auf Basis von Projektion und Tiefendatenerkennung vor. Konkret geht es um ein Assistenzsystem für manuelle Montageprozesse, das im vom BMWi geförderten Forschungsprojekt motionEAP entwickelt wurde. Neben der technischen Umsetzung werden die pädagogischen, psychologischen und ethischen Aspekte für die Nutzung dieses Assistenzsystems diskutiert. Ein besonderer Aspekt bezieht sich darauf, wie solche Systeme dazu beitragen können, leistungsgewandelte und leistungsgeminderte Menschen besser in die Arbeitswelt zu integrieren.

Ein zweites Szenario bezieht sich auf die industrielle Servicerobotik am Beispiel der Kleinteilemontage. André Hengstebeck, Kirsten Weisner, Jochen Deuse, Jürgen Rossmann und Bernd Kuhlenkötter berichten im Kontext des BMWi-geförderten Forschungsprojekts MANUSERV über die Entwicklung einer webbasierten Planungsumgebung, welche die Potenziale industrieller Robotersysteme mit den spezifischen Anforderungen manueller Arbeitssysteme und -prozesse verknüpft. Im konkreten Anwendungsfall wird die Montage eines Einbauradios mit Touch-Display, das auf Basis einer konkreten Produktspezifikation des Kunden hergestellt wird, betrachtet. Dabei werden die Potenziale der Nutzung von Leichtbau-Servicerobotern untersucht. 
Im BMBF-geförderten Forschungsprojekt SmARPro (Smart Assistance for Humans in Production Systems) wird ein System entwickelt, das über einheitliche und standardisierte Schnittstellen Daten aller umgebenden Systeme erfasst und diese in der SmARPro-Plattform zu kontextsensitiven Informationen aufbereitet. Diese werden dem Mitarbeiter über Wearables wie beispielsweise Datenbrillen, Smart Watches, Smartphones oder Tablets angezeigt. Benedikt Mättig, Jana Jost und Thomas Kirks beschreiben Anwendungsfälle im Bereich der Logistik, wo beispielsweise Kommissionierern und Wareneingangskontrolleuren ihren jeweiligen Rollen und der Situation angepasste Informationen in ihre Datenbrillen eigeblendet werden.

Mit den Auswirkungen von Industrie 4.0 auf die Arbeit in einer Weberei beschäftigen sich Mario Löhrer, Jacqueline Lemm, Daniel Kerpen, Marco Saggiomo und Yves-Simon Gloy im Kontext der vom BMBF geförderten Nachwuchsforschungsgruppe SozioTex an der RWTH Aachen. In dieser Nachwuchsforschergruppe wurde eine interdisziplinäre Methode zur Entwicklung soziotechnischer Assistenzsysteme in der textilindustriellen Produktionsarbeit entwickelt. Der konkret beschriebene Anwendungsfall bezieht sich auf eine Weberei für technische Textilien, wo auf industriellen Webmaschinen beispielsweise Sicherheitsgurte für die Automobilbranche hergestellt werden. Dort wird ein Assistenzsystem zur Arbeitsunterstützung untersucht, das sich auf ein Tablet als mobiles Endgerät in Verbindung mit Augmented Reality (AR) stützt.

Roman Senderek präsentiert Ergebnisse des vom BMBF geförderten Verbundprojekts ELIAS (Engineering Lernförderlicher Industrieller Arbeitssysteme) anhand der Anwendungsfälle der HELLA KGaA Hueck \& Co. und der FEV GmbH, zweier der größten Unternehmen aus dem deutschen Automotive-Sektor. In dem Projekt wird ein Konzept entwickelt, das das Lernen im Prozess der Arbeit in bestehende oder zukünftige Arbeitssysteme integriert. Die beiden Beispiele zeigen, dass Maßnahmen zur betrieblichen Weiterbildung eine immer größere Bedeutung gewinnen. Beide Unternehmen reagieren auf diesen Wandel mit dem verstärkten Einsatz des arbeitsnahen Lernens, in Form unterschiedlicher Lernlösungen.

„Assistenz und Wissensvermittlung am Beispiel von Montage- und Instandhaltungstätigkeiten“ ist das Thema von Carsten Ullrich, Axel Hauser-Ditz, Niklas Kreggenfeld, Christopher Prinz und Christoph Igel. Im Verbundprojektes APPsist, das vom BMWi gefördert wird, geht es um die Entwicklung mobiler, kontextsensitiver und intelligentadaptiver Assistenzsysteme, welche die Mitarbeiter beim Wissens- und Kompetenzerwerb in der Interaktion mit Maschinen auf dem Shopfloor unterstützen. Im konkreten Anwendungsszenario soll der Wechsel eines Werkstoffes in einer teilautomatisierten Montagelinie durch eine angelernte Montagekraft mithilfe von Assistenz durchgeführt werden. Durch die Assistenzsysteme sollen also an- oder ungelernte Mitarbeiter/Innen dazu befähigt werden, komplexere Prozesse selbstständig und effizient durchzuführen.

Das Projekt InnoCyFer wird vom BMWi gefördert. Susanne Vernim, Christiane Dollinger, Andreas Hees und Gunther Reinhart berichten über die Entwicklung eines Planungsund Steuerungssystems für eine autonome, auf cyber-physischen Systemen basierende Produktion. Die Produktionsplanung und -steuerung wird hier über einen sogenannten 
bionischen Scheduler, der auf einem Ameisenalgorithmus basiert, mit dem physischen Produktionssystem gekoppelt. Der Produktionsplaner wird über die Planungsvorschläge oder Steuerungsentscheidungen des bionischen Schedulers informiert und kann bei Bedarf die gewünschte Möglichkeit auswählen oder bewerten. Dadurch kann er steuernd eingreifen und zu jeder Zeit Produktionsentscheidungen durch eine transparente Aufbereitung nachvollziehen.

Ebenfalls vom BMWi gefördert wird ReApp - wiederverwendbare Roboterapplikationen für flexible Roboteranlagen; dieses Projekt hat Werkzeuge und Modelle für die Entwicklung wiederverwendbarer Softwarebausteine (Apps) für Roboter zum Gegenstand. Im Beitrag von Ulrich Reiser, Uwe Müller, Mike Ludwig Mathias Lüdtke und Yingbing Hue wird die Bestückung von Durchsteckbauelementen auf Leiterplatten als Szenario betrachtet. Dieser Prozess ist, trotz generell hoher Automatisierungsgrade bei der Bestückung von Leiterplatten, immer noch zum großen Teil Handarbeit. Als Lösung wurde hier ein automatischer Lötkolben mit Lötdrahtvorschub als Bestandteil einer mobile Einheit konzipiert, die mit einem Leichtbauroboter, allen nötigen Steuereinheiten und auch Sicherheitseinrichtungen ausgestattet werden kann.

Alle diese Praxisbeispiele werden nach den am Anfang dieses Kapitals dargestellten Kriterien bzw. Beschreibungsdimensionen dargestellt. Dadurch wird es möglich, die unterschiedlichen Szenarien nach einheitlichen Maßstäben zu vergleichen und einen Eindruck über mögliche Implikationen und Auswirkungen von konkreten Implementierungen von Industrie $4.0 \mathrm{zu}$ gewinnen.

\subsection{Aspekte und Perspektiven zukünftiger Arbeitssystemgestaltung}

Neben den Praxis-Szenarien aus aktuellen Forschungs- und Entwicklungsprojekten werden einige übergreifend bedeutsame Aspekte und Perspektiven von Industrie 4.0 in weiteren Beiträgen dargestellt.

Wilhelm Bauer, Sebastian Schlund und Tobias Strölin stellen zunächst das arbeitsplatznahe Beschreibungsmodell für die Arbeitswelt Industrie 4.0 vor, das auch schon den Darstellungen der Praxis-Szenarien im Hauptteil dieses Bandes zu Grunde lag. Dieses Modell wird im Rahmend des GMA-Fachausschusses 7.22 „Arbeitswelt Industrie 4.0“ der VDI/ VDE-Gesellschaft Mess- und Automatisierungstechnik aufgegriffen und weiterentwickelt.

Wenke Apt und Marc Bovenschulte stellen die Frage der Arbeitsgestaltung für Industrie 4.0 in den Kontext des demografischen Wandels und diskutieren vor diesem Hintergrund die Relevanz von Assistenz- und Tutorensystemen in der Arbeitswelt. Apt und Bovenschulte plädieren dafür, zur Bewältigung des demografischen Wandels Systeme zur technischen Unterstützung zu entwickeln, die das Ziel einer befähigenden Digitalisierung anstelle einer substituierenden Automatisierung haben.

Ein Leitbild von sozialer, human- und kommunikationsorientierter Produktion und Logistik steht im Zentrum des Beitrags von Hartmut Hirsch-Kreinsen, Michael ten 
Hompel, Peter Ittermann, Johannes Dregger, Jonathan Niehaus, Thomas Kirks und Benedikt Mättig. Zentral für eine solche Entwicklungsperspektive von Arbeit sind Tätigkeiten mit weitgehenden Gestaltungsmöglichkeiten und der Nutzung intelligenter Assistenzsysteme, vollständige Gesamtaufgaben, Lernförderlichkeit und neue Formen der Selbstorganisation bei dezentraler Steuerung.

Das viele Beiträge dieses Bandes prägende Thema der lernförderlichen Arbeits- und Organisationsgestaltung wird von Thomas Mühlbradt, Peter Kuhlang und Thomas Finsterbusch ausführlich und im Zusammenhang diskutiert. Die Autoren betrachten Lernförderlichkeit als Kernelement einer unternehmerischen Strategie, die darauf abzielt, systematisch intellektuelles Kapital in den drei Dimensionen Human-, Struktur- und Beziehungskapital zu entwickeln.

Eine wesentliche Funktion im Kontext von Industrie 4.0 besteht darin, aus einer großen Menge an Daten Grundlagen für gute unternehmerische Entscheidungen zu gewinnen. Anne Meyer, Stefan Zander, Rico Knapper und Thomas Setzer präsentieren daher das Konzept der Decision Support Pipeline als Vision einer datengetriebenen System- und Werkzeuginfrastruktur. Sie demonstrieren Anwendungen dieses Konzepts in den Bereichen Logistik, Produktionsplanung und Predictive Maintenance.

Die völlig zu Unrecht selten thematisierte Frage nach Gerechtigkeit in flexiblen Arbeitsund Managementprozessen stellen Gregor Engels, Günter W. Maier, Sonja K. Ötting, Eckhard Steffen und Alexander Teetz. Während Gerechtigkeitswahrnehmungen bisher fast ausschließlich in der Mensch-Technik-Interaktion untersucht wurden, wird zunehmend überlegt, wie Prinzipien der Gerechtigkeit auch in die Mensch-System-Interaktion übertragen werden können. Dies ist eine der Fragestellungen, die im NRW Fortschrittskolleg „Gestaltung von flexiblen Arbeitswelten - Menschen-zentrierte Nutzung von CyberPhysical Systems in Industrie 4.0“ behandelt werden.

Open Access Dieses Kapitel wird unter der Creative Commons Namensnennung 4.0 International Lizenz (http://creativecommons.org/licenses/by/4.0/deed.de) veröffentlicht, welche die Nutzung, Vervielfältigung, Bearbeitung, Verbreitung und Wiedergabe in jeglichem Medium und Format erlaubt, sofern Sie den/die ursprünglichen Autor(en) und die Quelle ordnungsge-mäß nennen, einen Link zur Creative Commons Lizenz beifügen und angeben, ob Änderungen vorgenommen wurden.

Die in diesem Kapitel enthaltenen Bilder und sonstiges Drittmaterial unterliegen ebenfalls der genannten Creative Commons Lizenz, sofern sich aus der Abbildungslegende nichts anderes ergibt. Sofern das betreffende Material nicht unter der genannten Creative Commons Lizenz steht und die betreffende Handlung nicht nach gesetzlichen Vorschriften erlaubt ist, ist für die oben aufgeführten Weiterverwendungen des Materials die Einwilligung des jeweiligen Recht-einhabers einzuholen.

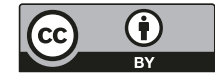

